\title{
Diabetic diagnose test based on PPG signal and identification system
}

\author{
Hadis Karimipour ${ }^{1}$, Heydar Toossian Shandiz ${ }^{1}$, Edmond Zahedi ${ }^{2}$ \\ ${ }^{1}$ School of Electrical Engineering, Shahrood University of Technology, Shahrood, Iran; ${ }^{2}$ School of Electrical Engineering, Sharif \\ University of technology, Tehran, Iran. \\ Email: $\underline{\text { h karimipour@yahoo.com; zahedy@sharif.edu }}$
}

Received 11 May 2009; revised 29 June 2009; accepted 6 July 2009.

\begin{abstract}
In this paper, photoplethysmogram (PPG) signals from two classes consisting of healthy and diabetic subjects have been used to estimate the parameters of Auto-Regressive Moving Average (ARMA) models. The healthy class consists of 70 healthy and the diabetic classes of 70 diabetic patients. The estimated ARMA parameters have then been averaged for each class, leading to a unique representative model per class. The order of the ARMA model has been selected as to achieve the best classification. The resulting model produces a specificity of $\% 91.4$ and a sensitivity of, $\% 100$. The proposed technique may find applications in determining the diabetic state of a subject based on a non-invasive signal.
\end{abstract}

Keywords: PPG Signal; Diabetic; Identification; ARMA Model

\section{INTRODUCTION}

Diabetes has been recognized as fourth leading cause of death in developed countries. Prediction based on recorded data in health centers worldwide shows that it is reaching epidemic proportions in many developing and newly industrialized nations [1].

When the body has difficulty regulating the amount of glucose in the blood stream Diabetes Mellitus has been occurred. Rising of the blood sugar level which will lead to hyperglycemia or hypoglycemia is due to the glucose accumulates in the bloodstream [2-3]. Glucose level above $150-160 \mathrm{mg} / \mathrm{dl}$ for long time poses significant health risk with possible long lasting effect [4]. Easy, low cost and on time recognizing diabetic with simple method and portable technology for the primary care and community-based clinical settings is the main goal of researchers in this area. The PPG technology has been used in a wide range of commercially available medical devices for measuring oxygen saturation, blood pressure and cardiac output [5]. Due to change in glucose level, the amount of blood volume in the figure changes, this variation can be measured by PPG. When a fixed source of infrared radiation is used, the variation of blood volume act as a phototransistor and the receive signal is changed. This is why we use the PPG signal for recognizing the diabetic. In this work by filtering on photoplethysmography (PPG) signal and estimate ARMA model for healthy and patient, a method for recognizing diabetic is proposed. Field data shows this method work properly.

\section{METHODOLOGY}

Identification systems methods are the best way for finding mathematic description of a black box. Figure 1 shows such a system in which only input and output terminals are introduced.

If there is no a noise source inside the system or measurement input and output are noise free, the number of unknown parameters in the system can determine the number of required measurements. As the real systems mathematically describe with finite parameter, everybody can determine the model easily. Perturbation in the system parameter and noise in the measurements lead to parameter estimation of the system.

Figure 2 shows the flow chart of each system identification method. The prior knowledge is used in all part of the model calculation. Based on the prior knowledge the experiment is set up to produce the data. The data have to be as much as informative. The observer adjusts the frequency content and amplitude of the input signals in the system with external input or chose the probability density function (PDF) in the system with unknown input.

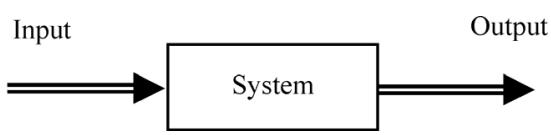

Figure 1. A black box system. 


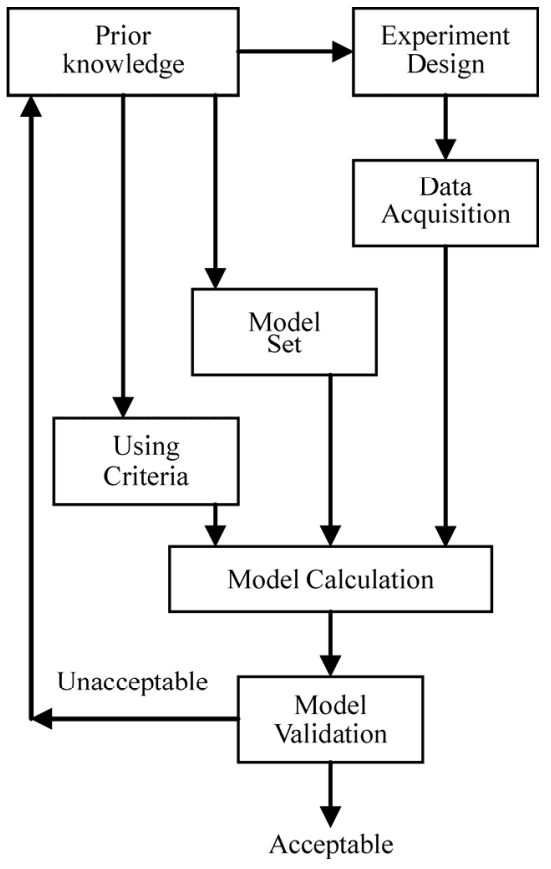

Figure 2. System identification flow chart.

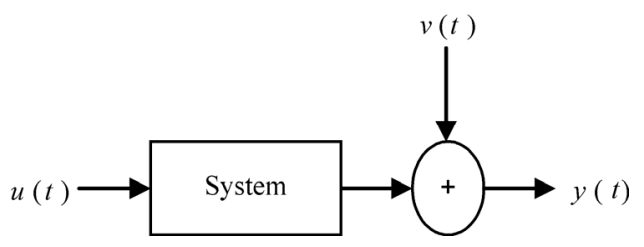

Figure 3. Modeling disturbance.

Choosing model set is second step in system identification based on prior knowledge. Different set of model is chosen, by considering number of input and output, linearity or nonlinearity, coupling or uncoupling between inputs and outputs, discrete or continues in time, frequency domain or time domain, application of calculated model for simulation, simplifying, controlling or inverse engineering.

Figure 3 shows a linear, time invariant, single input and output (SISO) model in which disturbance in system parameters and noise in measurement are modeled as additive in output [6]. This kind of model set is described as:

$y(t)=\sum_{k=1}^{n_{a}} a_{k} y(t-k)+\sum_{k=0}^{n_{b}} b_{k} u(t-k)+v(t)$

In which $y(t)$ is output and $u(t)$ is input, $v(t)$ is disturbance which is modeled as:

$$
v(t)=\sum_{k=0}^{n_{c}} c_{k} e(t-k)
$$

where, $e(t)$ is white noise .

The produced data are used to calculate the coefficients $a_{i}, b_{i}$ and $c_{i}$ of the model.

Next step is using criteria. Least square mean error (LSE) is used as the positive and negative error is the same and small error becomes smaller and big error become much bigger. The model rewrite as follow:

$$
y(t)=\varphi(t) \theta
$$

In which data are put in $\varphi(t)$ and $\theta$ contain all unknown parameter.

As the data is mixed with the measurement noise only an estimation of $\theta$ can be calculated. There are many criteria, we chose minimization of error between real output and model output as follow:

$$
V\left(\theta, Z^{N}\right)=\frac{1}{N} \sum_{t=1}^{N} e^{2}(t)
$$

In which $Z^{N}$ is input - output measured data and

$$
e(t)=y(t)-\varphi(t) \theta
$$

Therefore

$$
\hat{\theta}=\arg \min _{\theta} V\left(\theta, Z^{N}\right)
$$

The last step in model estimation is model validation. If in some sense the output of the model is fitted on the output of the system the estimated model is accepted otherwise the process is repeated again.

Model in Figure 3, is called autoregressive moving average extra input (ARMAX). Autoregressive means $y(t)$ depend on previous amount of it. X stand for external input $u(t)$ and MA stand for moving average refer to last term in Figure 3.

In the following subsections the proposed method is explained.

\subsection{Model Selection}

As there is no exact information about causes which affected PPG signal, ARMA model is used to model healthy and patient. The output of such system called time series. Figure 4 shows such systems.

In order to modeling the system mathematically equation 1 reduced to 7 :

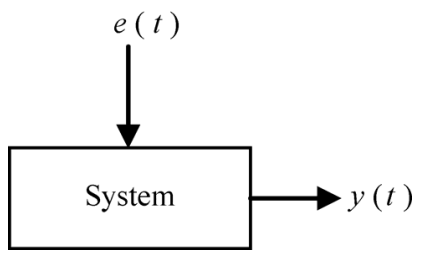

Figure 4. Modeling time series. 


$$
y(t)=\sum_{k=1}^{n_{a}} a_{k} y(t-k)+\sum_{k=0}^{n_{c}} c_{k} e(t-k)
$$

In which $y(t)$ is PPG signal as output and white noise $e(t)$ whit zero mean and variance $\lambda$ as input. This is like other time series analysis, such as vocal system, weather system, in which an effect without cause is in our hand.

The process of finding model is as follow:

Each patient and healthy data is used to calculate an ARMA model individually then the average of all models for each group is evaluated as the ARMA model for that category. After testing polynomials with deference dimensions, it was found that the $n_{a}=15$ and $n_{c}=11$ for patient model's parameter and $n_{a}=15$ and $n_{c}=10$ for healthy model's parameter, give the best result.

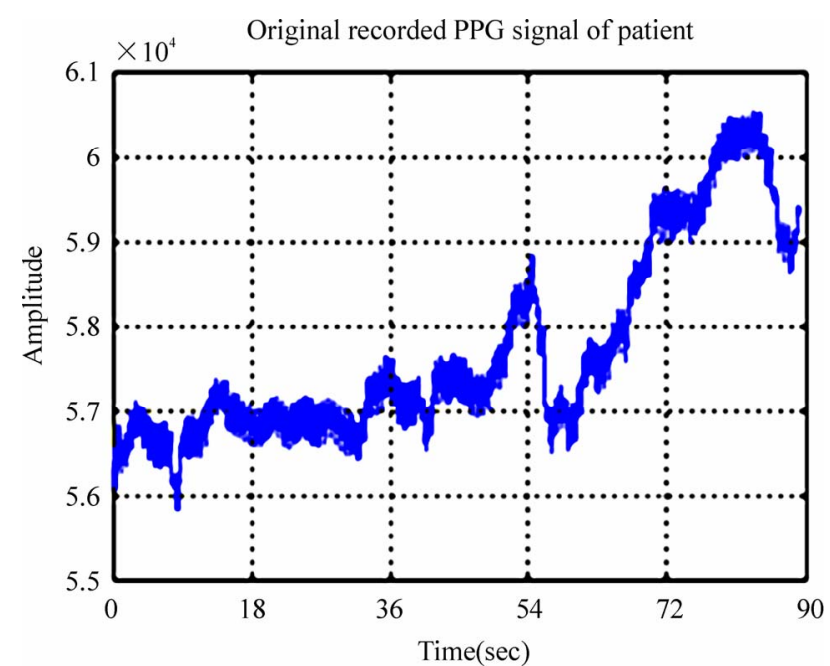

(a)

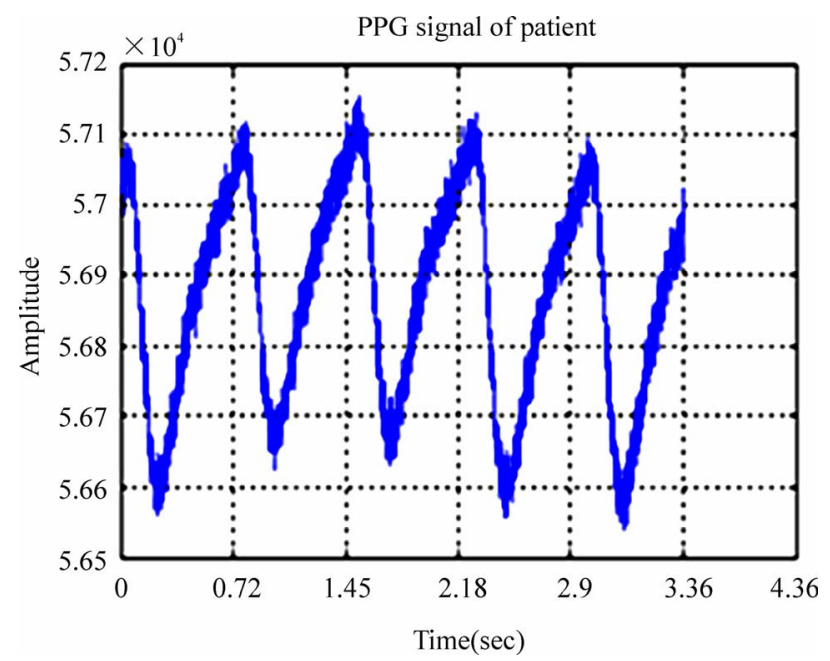

(b)

Figure 5. (a) recorded PPG signal and (b) one stable part with 1000 sample of it.

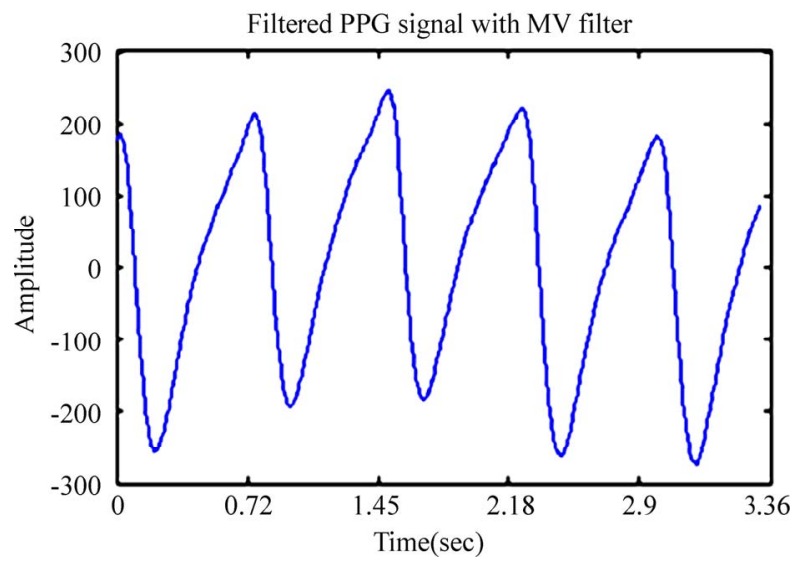

Figure 6. Result of applying moving average on healthy PPG signal.

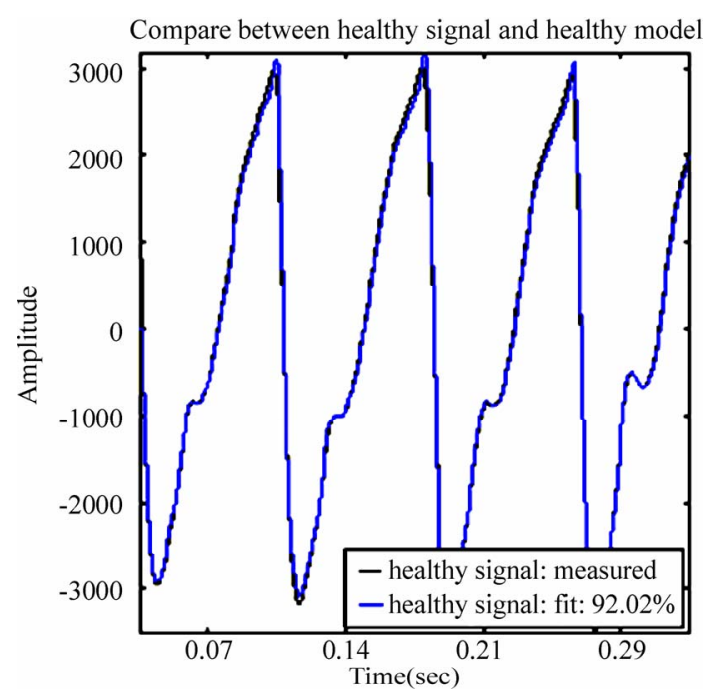

(a)

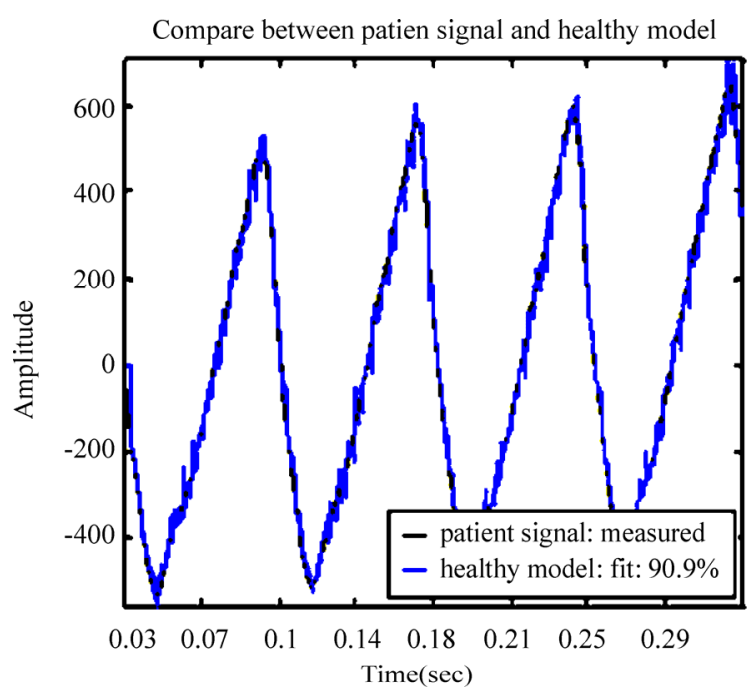

(b)

Figure 7. Result of applying (a) Patient PPG signal and (b) healthy PPG signal on the healthy model.

Openly accessible at http://www.scirp.org/journal/JBISE/ 


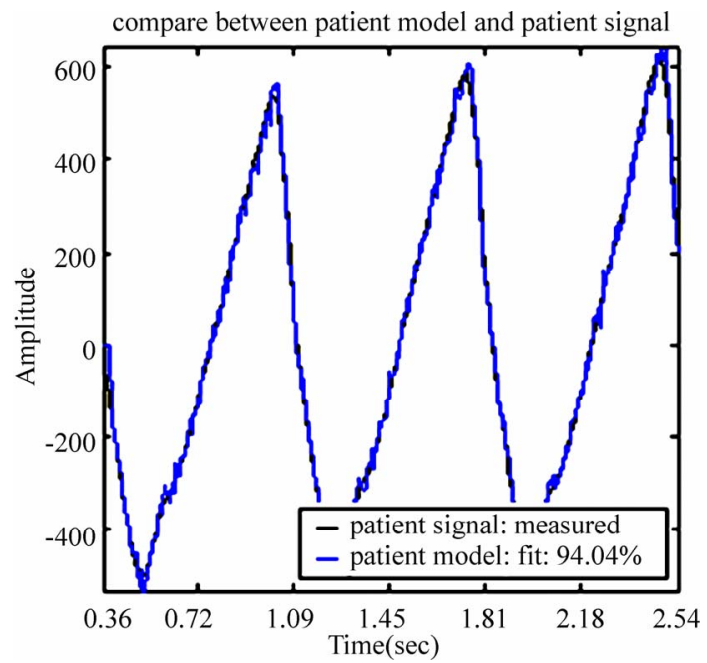

(a)

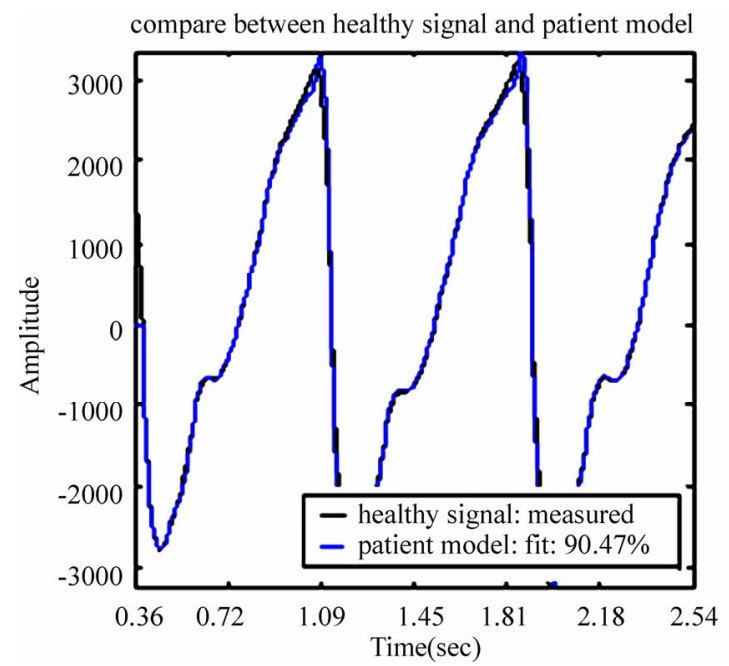

(b)

Figure 8. Result of applying (a) Patient PPG signal and (b) healthy PPG signal on the patient model.

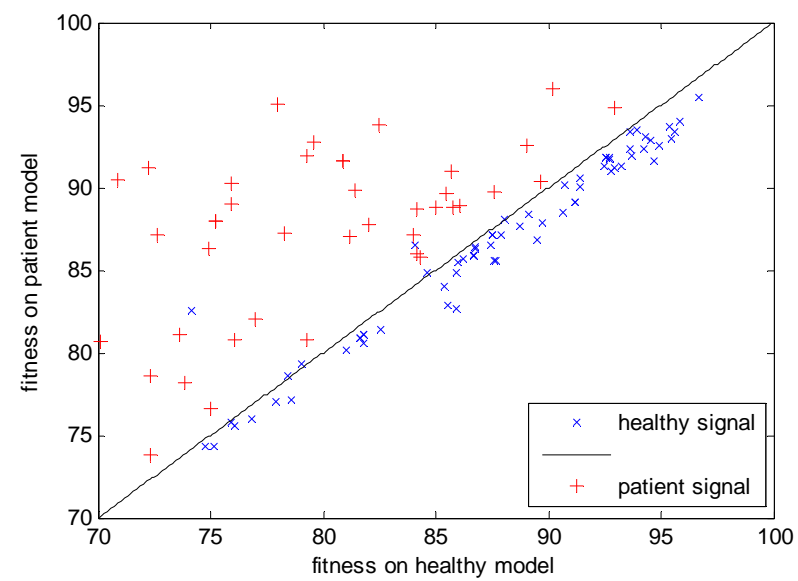

Figure 9. Result of applying PPG signal on the patient and healthy model.

\subsection{Experimental Setup}

There are two array groups of PPG signals. Pathologic arrays contain data from all subject tested in the hospital who were diabetic (39-64 years ages). Healthy arrays contain all data from healthy subjects (22-52 years old). Figure 1 shows a recorded PPG signal for a diabetic patient .

In each file, the only variable is a (50x24750) which is the raw PPG data:

- Each row is one particular lead.

- First column: subject number: Sb

- Second column: lead number: Ld

- Third column: Age

- Fourth column up to end: raw PPG data

- The length of the files has been limited to $90 \mathrm{sec}$ (sampled at $275 \mathrm{~Hz}$, this gives 24750 sample points)

- The number of rows (records) has been limited to 50 per file to limit file size

- The format of the data is uint32 to save on space.

\subsection{Criteria and Model Validation}

The MATLAB identification toolbox is used to calculate model parameter. The LSE is used as criteria and output matching as model validation.

\section{PRACTICAL RESULTS}

Figure 5 shows a sample of PPG signal of healthy and patient which is recorded in a hospital.

The additive noise corrupts the PPG signals. Moving average filter is used to remove disturbance from signal. Figure 6 shows output of the filter.

Figure 7 and 8 show the result of applying Healthy and patient PPG signal on estimated model for healthy and patient samples.

There are two areas in Figure 9, if data fit on healthy model better than patient model, there is a point under the line (subject is healthy) and inverse the point on the line shows possibility of wrong classification.

As Table 1 shows the proposed method can calcify healthy person and patient by $100 \%$ and $94 \%$, respectively.

Simulation result shows that, specificity, sensitivity, negative predictive value (NPV) and positive predictive

Table 1. Contingency table.

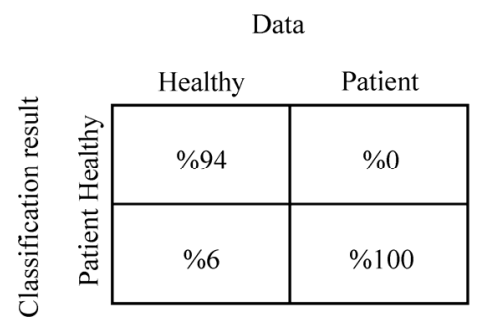


value (PPV) is \% 91.4, \% 100, \% 100, and \% 92.1 respectfully.

\section{CONCLUSIONS}

The proposed technique using non-invasive PPG signals is able to separate healthy subjects from patients using an ARMA model. One potential application of these models could be to estimate the state of diabetes of patients and eventually be used in controlling the amount of insulin needed to be injected to diabetic people.

\section{ACKNOWLEDGEMENTS}

The authors gratefully acknowledge the PPG signals kindly provided by the Faculty of Engineering and Built Environment, University Kebangssan Malaysia.

\section{REFERENCES}

[1] D. Gan, editor, (2003) Diabetes atlas, 2nd Edition, Brussels: International Diabetes Federation,
http://www.eatlas.idf.org/webdata/docs/Atlas\%202003-S ummary.pdf.

[2] H. L. Wee, H. K. Ho, and S. C. Li, (2002) Public awareness of diabetes mellitus in singapore, J .Singapore Med 43(3), 128-134.

[3] Cesar Carlos Romanillos Palerm, (2003) Drug infusion control: An extended direct model reference adaptive control strategy, PhD thesis, Rensselaer Polytechnic Institute, Troy, New York.

[4] V. Carmen Doran, H. Nicolas Hudson, T. Katherine, J. Moorhead, Geoffrey Chase, M. Geoffrey Shaw, and E. Chris Hann, (2004) Derivative weighted active insulin control modelling and clinical trials for ICU patients, Elsevier ju, Medical Engineering \& Physics.

[5] V. K. Jayasree, T. V. Sandhya, and P. Radhakrishnan (2008) Non-invasive studies on age related parameters using a blood volume pulse sensor, Measurment Science Reveiw, 8(4), Section 2.

[6] L. Ljung, (1999) System identification: theory for user, Second edition, Prentice Hall. 